Research Article

\title{
Risk Cloud Model for Evaluating Nautical Navigational Environments
}

\author{
Lijia Chen (D) $^{1}$ and Yanfei Tian ${ }^{2,3}$ \\ ${ }^{1}$ School of Navigation, Wuhan University of Technology (WUT), Wuhan 430063, China \\ ${ }^{2}$ School of Naval Architecture and Maritime, Zhejiang Ocean University (ZJOU), Zhoushan 316022, China \\ ${ }^{3}$ Hubei Key Laboratory of Inland Shipping Technology, Wuhan 430063, China
}

Correspondence should be addressed to Yanfei Tian; feifei_whut@163.com

Received 10 September 2020; Revised 28 February 2021; Accepted 17 March 2021; Published 1 April 2021

Academic Editor: Luigi Rodino

Copyright $\odot 2021$ Lijia Chen and Yanfei Tian. This is an open access article distributed under the Creative Commons Attribution License, which permits unrestricted use, distribution, and reproduction in any medium, provided the original work is properly cited.

\begin{abstract}
Uncertainty makes the risk evaluation of complex water transportation systems (WTSs) a difficult task. To achieve reasonable results while accounting for uncertainty, the risk evaluation of nautical navigational environments (NNEts) is often based on classical cloud model theory. This study proposes the concept of a risk cloud model (RCM) for NNEt evaluation and uses a fuzzy statistics-based computational approach to obtain the RCM parameters. As a case study, the proposed RCM method was applied to the risk evaluation of the Qiongzhou Strait. The performance of the proposed method was compared to those of a fuzzy theorybased method and an earlier proposed simplified algorithm. The results of the case study demonstrated the effectiveness of the proposed method along with several key advantages. First, the method could deal with uncertainty, take advantage of multichannel information, and evaluate risk features. Second, the RCM droplets intuitively displayed the qualitative and quantitative characteristics of risk levels, which facilitated understanding and analysis. Third, it showed a good sensitivity to ensure the refinement of evaluation results. The proposed method offered an improved approach to NNEt risk evaluation under uncertain conditions.
\end{abstract}

\section{Introduction}

The risk evaluation of a water transportation system (WTS) is a controversial research topic because of its complexity and other problems. It includes much uncertainty involving the fuzziness of experts' qualitative comments, randomness of observation experiments (process and results), incomplete description of the research object, and nonexclusive conversion model (or mapping) between variables. Such widespread uncertainty increases the difficulty of integrating qualitative and quantitative results for the WTS risk evaluation. The results may have insufficient accuracy and credibility because of the lack of objective observation data, subjectivity of expert judgements, improper evaluation method, or incompetence of the evaluator. Thus, uncertainty needs to be considered [1] to improve the reliability of the risk evaluation and to obtain reasonable and credible results.
In general, comprehensive evaluation can be observed as a multiple attribute decision-making (MADM) undertaking. This type of undertaking requires consideration of multiple attributes or synthesis of information from multiple sources to provide comprehensive evaluation(s) on concerned object(s), namely, choosing appropriate element(s) from the comment set to depict the object(s). As mentioned previously, uncertainty exists when performing a comprehensive evaluation. In decision-making, there are some approaches that deal with uncertainty. For example, Chen et al. used fuzzy terms and the technique for order preference by similarity to an ideal solution (TOPSIS) method from the perspective of MADM for decision-making and conducted in-depth research on theory and application of fuzzy terms and MADM methods [2-5]. Wey proffered an integrated approach based on the fuzzy Delphi method and dynamic network process to identify sustainable urban transportation 
planning strategies to improve quality of life [6]. In recent years, many theories and models for the risk analysis and evaluation of WTS under uncertainty have been developed for special application field. Ventikos et al. applied a seismicdriven model to calculate the probability of accident occurrence, and they derived results through a statistical analysis [7]. Cao et al. constructed probability-consequenceincluded risk matrix to evaluate the risk of accidents that caused injury to crew [8]. Mabroukito et al. proposed a specific methodology based on analytic hierarchy process (AHP) to analyse and assess operational risk within the port terminals at the RO-RO activity [9]. Sii et al. proposed a fuzzy-logic-based approach to evaluate risk in maritime engineering and applied the model to evaluate fire risk in an offshore support vessel [10]. Pak et al. used a fuzzy analytical hierarchy process to evaluate the importance of factors and rank the safety levels of targeted ports in Korea from a captain's perspective [11]. Tian et al. developed a risk evaluation model for nautical navigational environment (NNEt) based on the grey fixed weight cluster method and applied the model to Qiongzhou Strait case [12]. Liu et al. carried out safety assessment for inland waterway transportation with an extended fuzzy TOPSIS [13]. Wu et al. evaluated the effectiveness of maritime safety control in different navigation zones along the Yangtze River using a spatial sequential DEA model [14].

The abovementioned methods have certain advantages. For example, depending on the complete dataset, the TOPSIS or DEA can directly perform calculations on the multidimensional data to determine decision results; probability-statistics-based approaches focus on overcoming the challenges of randomness, whereas other methods focus on overcoming the difficulties caused by fuzziness. As mentioned previously, uncertainty lies in many aspects. The mentioned approaches do not completely overcome the difficulties caused by uncertainty or reflect the characteristics of uncertainty. A definitive, reasonable, and credible evaluation requires an accurate mapping of the qualitative and quantitative variables, and it must also capture the essence of qualitative knowledge and uncertainty, both in terms of fuzziness and randomness. Thus, a new more appropriate method of risk evaluation should be developed based on the combination of new and existing theories, methods, etc.

$\mathrm{Li}$ et al. proposed a membership cloud model that combines the basic principles of probability theory and fuzzy set theory for application in the field of intelligent control $[15,16]$. Thereafter, the cloud model has been widely applied to the comprehensive evaluation of complex systems [17]. To account for randomness, fuzziness, and incompatibility, Wang et al. coupled a novel multidimensional cloud model with connection number theory to evaluate slope stability [18]. Dawei et al. used extension cloud theory to develop a comprehensive evaluation model for power market operation efficiency [19]. Li presented a rough cloud theory-based approach for a modified failure mode and effects analysis considering uncertainty [20]. Lou reported an integrated decision-making method to evaluate product design schemes based on a cloud model and electroencephalography (EEG) data [21]. Liu et al. coupled a connection cloud model with extenics to analyse and evaluate water quality [22]. Lv et al. applied a cloud model to evaluate the status of a mobile welding robot driven by a fuel cell hybrid power system [23] due to its abilities. Yang et al. reported a hesitant cloud model to quantitatively evaluate human-related risk along the $21^{\text {st }}$ Century Maritime Silk Road [24].

Thus, a cloud model is a powerful tool that integrates fuzziness and randomness to map qualitative and quantitative information together [25]. Knowledge representation and reasoning methods based on cloud models can completely express the fuzziness and randomness of uncertainty, and cloud models can be used to resolve missing information while gathering data [26]. These features make the comprehensive evaluation of complex systems suitable.

In this study, the classical cloud model theory was applied to the risk evaluation of NNEt to take full advantage of uncertain information and to obtain rational and credible results. The main contributions are as follows:

(1) A risk cloud model (RCM) based on the classical principles of cloud model theory is proposed for risk evaluation

(2) A fuzzy statistics-based computational approach is introduced to obtain the RCM parameters

(3) A case study was performed to validate the proposed method and demonstrate its advantages

The remainder of this paper is organised as follows. The modelling process, including the concept and construction of the proposed RCM, for evaluation, is described in Section 2. The steps used for risk evaluation using the RCM are specifically explained in Section 3. An application case and the comparative analysis of the proposed method are presented in Section 4. Finally, the conclusions are presented in Section 5 .

\section{Risk Cloud Model: Concept and Construction}

2.1. Variable Settings. Typically, a language variable and/or a numerical variable are given in advance for qualitative and/ or quantitative evaluation.

In general, the judgements for qualitative evaluation are mostly linguistic phrases; for example, the linguistic term set (soft, moderate, and hard) is used to evaluate the hardness of materials, the linguistic term set (excellent, good, average, passing, and failing) is used to evaluate the performance of students, the linguistic term set (extraordinarily serious, serious, relatively serious, and general) is used to record and reflect accidental severity, the linguistic term set (infant, childhood, teenager, youth, prime, middle, and old) is used to measure the state of persons' age, and the linguistic term set (low, moderately low, moderate, moderately high, and high) is used to measure the risk of warehouse temperature or a complex environmental system.

The linguistic terms are called fuzzy concepts in view of fuzzy theory. Chen et al. $[27,28]$ studied the definition and principle of language terms and their applications in comprehensive evaluation from the perspective of MADM 
in a more in-depth way to avoid information loss. Other existing class researches on linguistic variable are conducted by Xiong [29] and Farhadinia [30].

Further, a numerical variable (quantitative variable) with a set domain of discourse/range is often used for quantitative evaluation. For example, the numerical variable-score $(s)$ with the range $[0,100]$ is used for quantitative evaluation of students' performance, and the numerical variable risk index $(r)$ with the nondimensional range $[0,1]$ or $[0,10]$ or $[0$, $100]$ is used for quantitative evaluation of the risk level.

During the risk evaluation, the qualitative and quantitative variables used to measure the risk need to be properly defined. In terms of the WTS evaluation analysis, there are also some frequently used language variables, whose values are linguistic terms. For example, the linguistic term set (extraordinarily serious, serious, relatively serious, and general) is used to characterize the severity of water traffic accidents, and the linguistic term set (low, moderately low, moderate, moderately high, and high) is applied to measure the risk level of complex transportation scenes. In addition, a numerical variable called risk index $(r)$ with the nondimensional range $[0,1]$ or $[0,10]$ or $[0,100]$ is used for quantitative evaluation.

According to the literature on WTS risk evaluation [31-34], five linguistic terms are typically used to qualitatively demarcate the risk level: low (L), moderately low (ML), moderate $(\mathrm{M})$, moderately high $(\mathrm{MH})$, and high $(\mathrm{H})$. In addition, a specially called numerical variable is typically used when qualitative evaluation is required. Similarly, a linguistic variable-comment $(\mathrm{cmt})$ was used in this study to indicate the risk level qualitatively, where $B$ is the set of five basic elements: $B=\{\mathrm{L}$ ML M MH H $\}$. Meanwhile, a numerical variable-score $(x)$ was used for quantitative evaluation, where $\left[\begin{array}{ll}x_{\min } & x_{\max }\end{array}\right]=\left[\begin{array}{ll}0 & 1\end{array}\right]$. Table 1 lists the underlying relationship between the two types of variables used for risk evaluation.

\subsection{RCM: Concept, Construction, and Definitions. Li et al.} proposed a mathematical definition for the cloud model [15]. Many cloud models have been developed, but the normal cloud model is the most commonly used [35]. Thus, the remainder of this paper will solely refer to the normal cloud model. A cloud model effectively integrates three variables to represent a qualitative concept in a quantitative manner: the expected value (Ex), entropy (En), and hyperentropy $(\mathrm{He})$ [36]. This is denoted by $C(\mathrm{Ex}, \mathrm{En}, \mathrm{He})$, where $\mathrm{Ex}$ is the distributed expectation of cloud droplets in the distribution domain, En is the uncertainty (or stochastic) measure of the qualitative concept, and $\mathrm{He}$ is the uncertainty measure of the randomness and fuzziness of entropy.

Based on these initial definitions and numerical characteristics of the cloud model, this study proposes an RCM for NNEt risk evaluation. The RCM is represented by $R\left(R_{1}, R_{2}, R_{3}\right)$, and it comprehensively depicts the risk level of a NNEt. $R_{1}$ represents the mathematical expectation of the numerical variable $x$, and thus, it is the most typical value used for quantitative evaluation. $R_{2}$ represents entropy and indicates the available range extending on both sides of $R_{1}$.
$R_{3}$ represents hyperentropy and indicates the level of certainty (i.e., degree of membership) about $x$. Thus, $R_{2}$ and $R_{3}$ can be used together to quantitatively reflect the uncertainty level of an evaluation. Note that $R\left(R_{1}, R_{2}, R_{3}\right)$ is the final result after all available information has been integrated. Thus, an RCM can be used for comprehensive evaluations. The key to developing an RCM is to determine $R_{1}, R_{2}$, and $R_{3}$. The computational approach to obtain the RCM parameters and steps of using the RCM for evaluation are provided in the following section.

2.3. Reference Scale Settings. The reference scale is used in risk evaluation to distinguish the risk level qualitatively and/ or quantitatively. Its settings are presented here. In the literature [37], a quantitative variable is regarded as a space holding all types of linguistic terms. Given the five linguistic concepts and domain of discourse $\left[\begin{array}{ll}y_{\min } & y_{\max }\end{array}\right]$ of a numerical variable $y$, the golden section search can be used to obtain the cloud model parameters [26]. In this study, the RCMs corresponding to five linguistic terms used for NNEt risk evaluation are referred to as benchmark RCMs. Table 2 lists the algorithms used in the literature [26] to determine the reference scales to calculate the characteristic parameters of the benchmark RCMs.

The hyperentropy of the middle cloud model $C_{0}$, corresponding to the third linguistic concept, is typically set to $0.005\left(\mathrm{He}_{0}=0.005\right)$ [25]. Table 3 lists the benchmark RCMs corresponding to the five linguistic terms based on the defined linguistic variable, comment $(\mathrm{cmt})$ and numerical variable, and score $(x)$, and using the algorithms in Table 2, the hyperentropy of the middle RCM $C_{\mathrm{M}}$ is set to 0.005 $\left(R_{3_{0}}=0.005\right)$. Figure 1 depicts the benchmark RCMs, with each containing 1000 droplets.

\section{Computational Approach for Risk Evaluation}

The computational approach used to obtain the RCM parameters is presented in this section.

3.1. Step 1: Selecting Evaluation Factors. As one of the subsystems of the WTS, the NNEt comprises many factors (e.g., wind, waves, currents, tides, fog, visibility, channel length, channel width, channel water depth, channel bend, shipping route crossing, ship traffic flow volume, ship traffic flow density, obstacles to navigation, and navigational aids). Table 4 lists the factors often considered in marine traffic safety (risk) studies. Several factors are commonly considered, including wind, currents, visibility, channel water depth, and traffic flow density. Individual factors are also considered depending on the time and location of the study area, such as the temperature for navigation during the hot season and the sea ice for Arctic navigation. According to the literature $[38,39]$, these factors can be classified into three categories (natural environment, channel condition, and traffic environment) or two categories (natural and artificial conditions). Table 4 also presents the categories of factors considered by scholars. A systematic analysis of the methods and results in the literature showed that the NNEt in general 
TAвLE 1: Relationship between linguistic and numerical variables.

\begin{tabular}{|c|c|c|c|}
\hline Type & Name & Symbol & Domain of discourse \\
\hline Linguistic & Comment & $\mathrm{cmt}$ & From $\mathrm{L}$ to $\mathrm{H}$ and holding five benchmark values: $\mathrm{L}, \mathrm{ML}, \mathrm{M}, \mathrm{MH}$, and $\mathrm{H}$ \\
\hline Numerical & Score & $x$ & {$\left[\begin{array}{ll}x_{\min } & x_{\max }\end{array}\right]=\left[\begin{array}{ll}0 & 1\end{array}\right]$} \\
\hline
\end{tabular}

TABLE 2: Golden section method-based algorithms.

\begin{tabular}{|c|c|c|c|c|c|}
\hline \multirow{2}{*}{$\mathrm{S} / \mathrm{N}(k)$} & \multirow{2}{*}{ Linguistic concepts } & \multirow{2}{*}{ Cloud model symbols } & \multicolumn{3}{|c|}{ Cloud model (characteristic parameters) } \\
\hline & & & Ex & En & $\mathrm{He}$ \\
\hline 1 & Concept 1 & $C_{-2}$ & $y_{\min }$ & $\mathrm{En}_{-1} / 0.618$ & $\mathrm{He}_{-1} / 0.618$ \\
\hline 2 & Concept 2 & $C_{-1}$ & $\mathrm{Ex}_{0}-0.382 \times\left(y_{\min }+y_{\max }\right) / 2$ & $0.382 \times\left(y_{\max }-y_{\min } / 6\right)$ & $\mathrm{He}_{0} / 0.618$ \\
\hline 3 & Concept 3 & $C_{0}$ & $y_{\min }+y_{\max } / 2$ & $0.618 \times \mathrm{En}_{+1}$ & Designated $\mathrm{He}_{0}$ \\
\hline 4 & Concept 4 & $C_{+1}$ & $\mathrm{Ex}_{0}+0.382 \times\left(y_{\min }+y_{\max }\right) / 2$ & $0.382 \times\left(y_{\max }-y_{\min } / 6\right)$ & $\mathrm{He}_{0} / 0.618$ \\
\hline 5 & Concept 5 & $C_{+2}$ & $y_{\max }$ & $\mathrm{En}_{+1} / 0.618$ & $\mathrm{He}_{+1} / 0.618$ \\
\hline
\end{tabular}

TABLE 3: Reference scale: benchmark RCMs corresponding to five linguistic terms.

\begin{tabular}{lcccc}
\hline S/N $(k)$ & & & \multicolumn{2}{c}{$\begin{array}{c}\text { Benchmark RCM (characteristic } \\
\text { parameters) }\end{array}$} \\
& Linguistic terms & Benchmark RCM symbols & $R_{1}$ & $R_{2}$ \\
\hline 1 & $\mathrm{~L}$ & $C_{\mathrm{L}}\left(=C_{-2}\right)$ & 0 & 0.1031 \\
2 & $\mathrm{ML}$ & $C_{\mathrm{ML}}\left(=C_{-1}\right)$ & 0.309 & 0.064 \\
3 & $\mathrm{M}$ & $C_{\mathrm{M}}\left(=C_{0}\right)$ & 0.5 & 0.039 \\
4 & $\mathrm{MH}$ & $C_{\mathrm{MH}}\left(=C_{+1}\right)$ & 0.691 & 0.008 \\
5 & $\mathrm{H}$ & $C_{\mathrm{H}}\left(=C_{+2}\right)$ & 1 & 0.064 \\
\hline
\end{tabular}

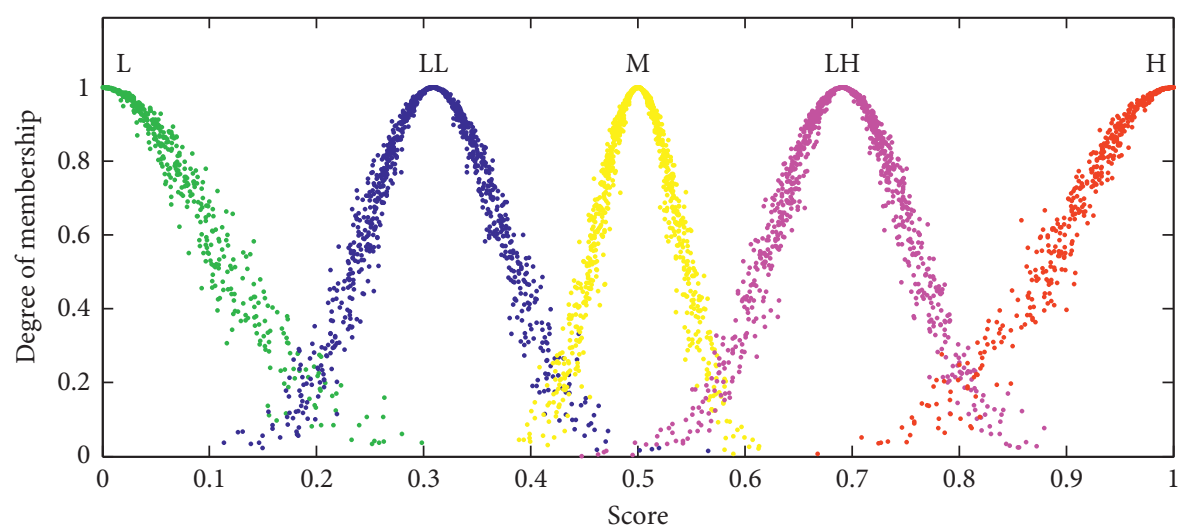

FIgURE 1: Reference scale: appearances of five benchmark RCMs.

or some of its constituent factors can be treated as analysis objects. The selected factors can then be used for risk evaluation.

3.2. Step 2: Initial Evaluation. In practice, the evaluation results are typically presented qualitatively because this helps facilitate intuitive understanding. Therefore, experts are asked to fill out a concise questionnaire to obtain a qualitative evaluation of a particular factor. Table 5 presents an example questionnaire, where an expert places a checkmark $(\checkmark)$, where appropriate, to indicate his/her evaluation of a particular factor.

3.3. Step 3: Conversion of Initial Evaluation to Risk Cloud Model. Because the RCM is a type of cloud model, the key step is the one-dimensional backward normal cloud generator algorithm [51-53]. The computational process is given as follows. 


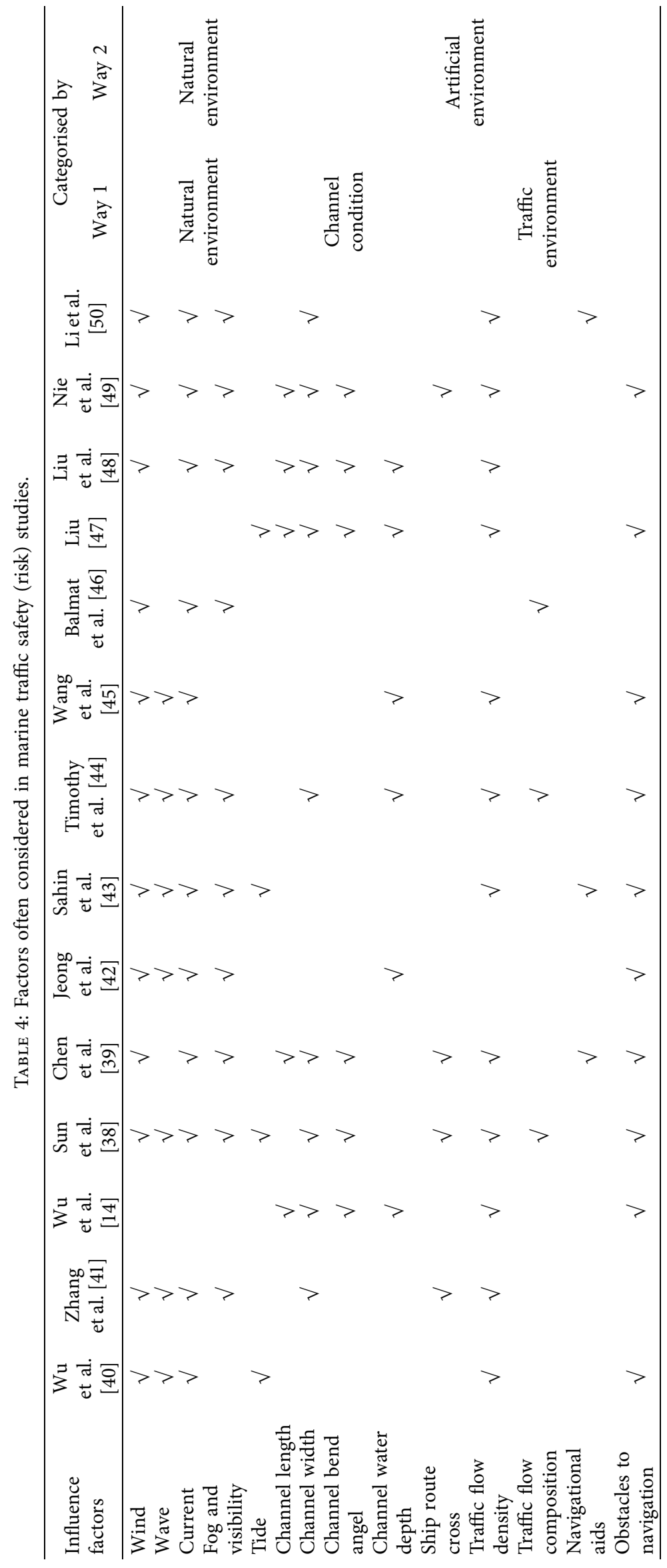


TABLE 5: Example questionnaire: the core part.

\begin{tabular}{llllll}
\hline \multirow{2}{*}{ Initial evaluation } & \multicolumn{5}{c}{ Linguistic terms } \\
& $\mathrm{L}$ & $\mathrm{ML}$ & $\mathrm{M}$ & $\mathrm{MH}$ & $\mathrm{H}$ \\
\hline A concerned factor & & & & & \\
\hline
\end{tabular}

3.3.1. Calculating the Expectation of the Sample Points. A qualitative evaluation can be transformed to a quantitative value as follows:

$$
x_{i}= \begin{cases}R_{1_{-2}}(=0.000), & \text { if } \mathrm{L} \text { is used by expert } i, \\ R_{1_{-1}}(=0.309), & \text { if } \mathrm{ML} \text { is used by expert } i, \\ R_{1_{0}}(=0.500), & \text { if } \mathrm{M} \text { is used by expert } i, \\ R_{1_{+1}}(=0.691), & \text { if } \mathrm{MH} \text { is used by expert } i, \\ R_{1_{+2}}(=1.000), & \text { if } \mathrm{H} \text { is used by expert } i .\end{cases}
$$

The arithmetic mean of the sample points $\left(x_{i}\right)$ can then be treated as the expectation $\left(R_{1}\right)$ :

$$
R_{1}=\frac{\sum_{i=1}^{n} x_{i}}{n}
$$

3.3.2. Calculating the Entropy of the Sample Points. The standard deviation of the sample points $\left(x_{i}\right)$ can be treated as the entropy $\left(R_{2}\right)$ :

$$
R_{2}=\sqrt{\frac{\sum_{i=1}^{n}\left(x_{i}-\bar{x}\right)^{2}}{n}}=\sqrt{\frac{\sum_{i=1}^{n}\left(x_{i}-\mathrm{Ex}\right)^{2}}{n}} .
$$

3.3.3. Calculating the Hyperentropy of the Sample Points. The entropy of each sample point can be calculated as follows:

$$
R_{2_{i}}=\sqrt{-\frac{\left(x_{i}-\bar{x}\right)^{2}}{2 \ln \mu_{i}}}=\sqrt{-\frac{\left(x_{i}-R_{1}\right)^{2}}{2 \ln \mu_{i}}},
$$

where $\mu_{i}$ is the degree of membership of a quantitative value $x_{i}$ and calculated using the fuzzy statistics method as follows:

$$
\mu_{i}=\frac{n_{i}}{n}
$$

where subscript $i$ refers to the $i$ - th initial evaluation, $n_{i}$ is the statistical occurrence times of $x_{i}$, and $n$ is the total experimental runs. Because the initial evaluation is based on an expert judgment, $n_{i}$ is equivalent to the number of experts evaluating a particular factor with the same linguistic term, and $n$ is equivalent to the total number of experts.

The standard deviation of entropy can then be treated as the hyperentropy $\left(R_{3}\right)$ as follows:

$$
R_{3}=\sqrt{-\frac{\sum_{i=1}^{n}\left(R_{2_{i}}-\overline{R_{2_{i}}}\right)^{2}}{n}}
$$

where $\overline{R_{2}}$ is the arithmetic mean of $R_{2_{i}}$.
3.4. Step 4: Plotting RCM Droplets. Given the characteristic parameters, the droplets of a cloud model can be produced using the forward cloud generator. The computational process is as follows:

Process name: computational process to plot RCM droplets.

Process input: characteristic parameters $\left(R_{1}, R_{2}, R_{3}\right)$ of an RCM and number of cloud droplets $(N)$.

Process output: sample point $\left(x_{j}\right.$; quantitative value) and degree of membership $\left(\mu_{j}\right)$ for $N$ cloud droplets:

(1) Generate a random number $\left(R_{2_{j}}\right)$ of a normal distribution: $R_{2_{j}} \sim N\left(R_{2}, R_{3}^{2}\right)$.

(2) Generate a random number $\left(x_{j}\right)$ of a normal distribution: $x_{j} \sim N\left(R_{1}, R_{2 j}^{2}\right)$.

(3) Let $x_{j}$ be a quantised value of the qualitative concept.

(4) Calculate the degree of membership $\left(\mu_{j}\right): \mu_{j}=e(-$ $\left.\left(\left(x-R_{1}\right)^{2} / 2 R_{2 j}^{2}\right)\right)$.

(5) Call the data point with the coordinate $\left(x_{j}, \mu_{j}\right)$ a cloud droplet, and

(6) Repeat steps (1)-(5) to generate $N(j=1,2,3, \ldots$, N) cloud droplets.

In an RCM, a cloud droplet is a data point with coordinates $\left(x_{j}, \mu_{j}\right)$, where $\mu_{j}$ is the degree of membership about $x_{j}$. $N$ cloud droplets of an RCM can be produced using the abovementioned computational process. Using the reference scale as background, the generated cloud droplets can be plotted in rectangular coordinates to visualise the RCM and facilitate analysis.

3.5. Step 5: Evaluation Results. The obtained RCM $R\left(R_{1}, R_{2}, R_{3}\right)$ can be used for a comprehensive evaluation of an evaluation factor. The characteristic parameters can be used to generate a quantitative evaluation. In general, the expectation $\left(R_{1}\right)$ depicts the central tendency of the expert judgements, and it is most often used to quantitatively indicate the risk level. The entropy $\left(R_{2}\right)$ and hyperentropy $\left(R_{3}\right)$ determine the discrete extent of the cloud droplets (e.g., breadth of the score distribution and thickness of cloud droplets), and they provide a quantitative measure of the uncertainty level of an evaluation.

A qualitative evaluation can be performed depending on the cloud droplet distribution in coordinates and by comparing the location of an RCM with those of the benchmark RCMs. If the obtained RCM coincides with a benchmark RCM, the exact linguistic term corresponding to the benchmark RCM is used for qualitative evaluation. If the obtained RCM is located elsewhere in the frame, it clearly corresponds to another linguistic term.

\section{Case Study and Comparative Analysis}

As a case study, the proposed RCM and computational approach were applied to the NNEt risk evaluation of the Qiongzhou Strait. The objectives of the case study were (1) to directly identify the risk level of evaluation factors and (2) to 
verify the feasibility and summarise the advantages of the proposed method.

4.1. Area of Interest. The Qiongzhou Strait is a narrow passage of water between the South China Sea and the Beibu Gulf $[54,55]$ at $20^{\circ} 09^{\prime} \mathrm{N}, 110^{\circ} 16^{\prime} \mathrm{E}$ [56]. The strait is about $70 \mathrm{~km}$ long and $30 \mathrm{~km}$ wide, with a maximum depth of $120 \mathrm{~m}$ [55] and minimum width of $18 \mathrm{~km}$ [57]. NNEt parameters for the Qiongzhou Strait, such as natural conditions, navigational channel layout, ship traffic flow, navigation obstacles, configuration of navigational aids, and safety management regulations for ships, have been comprehensively presented in the literature [58-63], and thus, are not repeated here. Figure 2 shows the specific area of interest for the case study.

4.2. Step 1: Selecting Evaluation Factors. As discussed in Section 3.1, a systematic analysis and literature review were conducted to select evaluation factors of the area of interest. Nine factors were selected by Sun [64] for risk evaluation based on fuzzy theory. Based on this, the same nine evaluation factors belonging to three categories were selected for analysis in this study, as listed in Table 6. This facilitated a comparison between the results of the present study and those mentioned in the literature.

4.3. Step 2: Initial Evaluation. For the initial evaluation, 29 expert questionnaires were collected in a previous study, consulting maritime authorities, ship crewmembers, port departments, shipping companies, and other maritime security departments [64]. Table 7 lists the initial evaluation obtained from the literature [64]. Table 8 presents the authors' own evaluation based on their understanding of the NNEt of the Qiongzhou Strait and professional knowledge of WTSs. In total, 30 data sources were obtained and utilised to generate RCMs of the evaluation factors.

4.4. Steps 3 and 4: RCMs and Plots of Droplets. Step 3 of the computational approach was employed to convert the initial qualitative evaluations into individual RCMs. Table 9 (column 1) lists the final RCMs of the evaluation factors using the proposed method. Next, step 4 was used to plot 1000 droplets of each RCM in rectangular coordinates, as shown in Figures 3-11 (the black dots).

4.5. Step 5: Evaluation Results. An RCM can be used to perform a comprehensive risk evaluation of a particular factor. Generally, the expectation $\left(R_{1}\right)$ depicts the central tendency of an expert's judgement, whereas the discrete extent of the cloud droplets as determined by the entropy $\left(R_{2}\right)$ and hyperentropy $\left(R_{3}\right)$ reflects the uncertainty of the evaluation process and results.

As an example, consider the RCM of factor "wind": $R(0.6391,0.1531,0.0605)$. For the quantitative evaluation, the expected value $R_{1}=0.6391$ denotes the mathematical expectation of score $(x)$, which indicates the risk level. The entropy

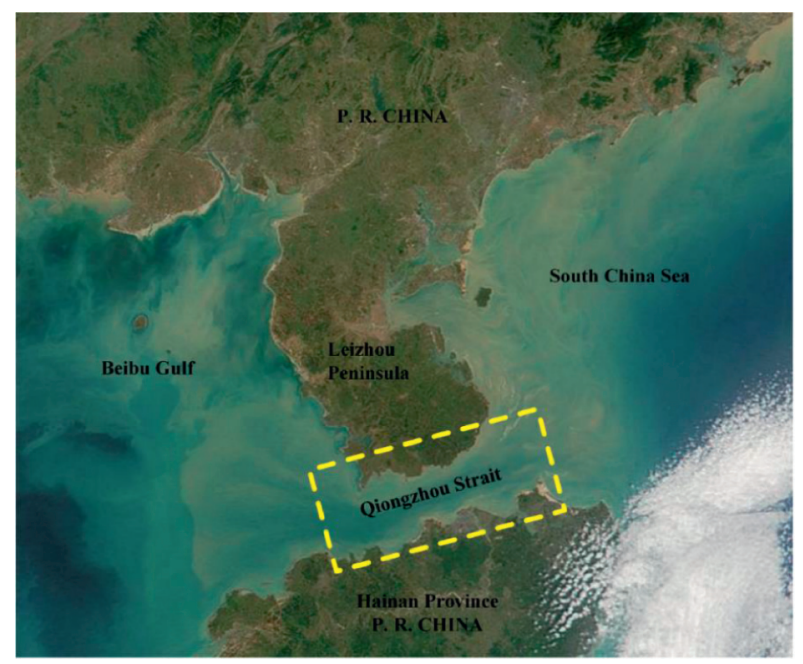

FIgURE 2: Location of the Qiongzhou Strait.

$R_{2}=0.1531$ denotes the measured uncertainty of the quantitative evaluation and reflects the stochastic characteristics of $x$; this implies that $x$ has a general distribution of $0.2-1.0$, as shown in Figure 3. The hyperentropy $R_{3}=0.0605$ indicates the degree of membership $(\mu)$ of $x$ and also that this RCM is thicker than the second or third benchmark clouds $\left(C_{\mathrm{ML}}\right.$ or $C_{\mathrm{MH}}$ with a hyperentropy of 0.008$)$. As shown in Figure 3, $R(0.6391,0.1531,0.0605)$ is very close to $C_{\mathrm{MH}}$. Thus, the qualitative evaluation can be intuitively expressed as slightly lower than the ML. Similarly, the quantitative and qualitative evaluations of the other selected factors can be performed based on their RCMs and corresponding appearances.

4.6. Comparative Analysis and Discussion. The results of the proposed RCM method were compared with those based on fuzzy theory to demonstrate its advantages.

4.6.1. Evaluation Based on Fuzzy Theory. In a previous study [64], fuzzy theory was used to evaluate risk $x$ based on the degree of membership and typical values corresponding to each fuzzy comment through a defuzzification process based on the weighted average. The evaluation of a selected factor is given by

$$
x_{\mathrm{FT}}=\sum_{k=1}^{5} s(B(k)) \cdot \mu(B(k)),
$$

where $x_{\mathrm{FT}}$ is the quantitative evaluation, $k$ is the sequence number $(\mathrm{S} / \mathrm{N}), B(k)$ is the $k$ - th benchmark linguistic term used for evaluation, $s(B(k))$ is the typical value of $B(k)$, and $\mu(B(k))$ is the degree of membership for $B(k)$.

From the reference scale, the expectation of the $k-$ th benchmark $\operatorname{RCM}(\operatorname{Ex}(k))$ was used to represent the typical value of $B(k)$ :

$$
s(B(k))=E x(k) .
$$

After the initial evaluation was obtained (Tables 7 and 8), $\mu(B(k))$ was derived using the fuzzy statistics method: 
TABLE 6: Study objects: chosen factors for analysis.

\begin{tabular}{|c|c|c|}
\hline Target system of interest & Categories (subsystems) & Concerned objects (chosen factors) \\
\hline \multirow{3}{*}{ NNEt of the Qiongzhou Strait waters } & Natural environment & $\begin{array}{c}\text { Wind } \\
\text { Current } \\
\text { Fog and visibility }\end{array}$ \\
\hline & Channel condition & $\begin{array}{l}\text { Channel width } \\
\text { Channel water depth } \\
\text { Shipping route cross }\end{array}$ \\
\hline & Traffic environment & $\begin{array}{l}\text { Ship traffic flow volume } \\
\text { Obstacles to navigation } \\
\text { Navigational aids }\end{array}$ \\
\hline
\end{tabular}

TABLE 7: Initial evaluation of the selected factors by 29 experts [64].

\begin{tabular}{|c|c|c|c|c|c|}
\hline \multirow{2}{*}{ Concerned objects } & \multicolumn{5}{|c|}{ Linguistic terms (for qualitative risk evaluation) } \\
\hline & $\mathrm{L}$ & ML & $\mathrm{M}$ & $\mathrm{LH}$ & $\mathrm{H}$ \\
\hline Wind & 0 & 0 & 13 & 14 & 2 \\
\hline Current & 7 & 18 & 3 & 1 & 0 \\
\hline Fog and visibility & 0 & 0 & 5 & 20 & 4 \\
\hline Channel width & 0 & 11 & 14 & 3 & 1 \\
\hline Channel water depth & 0 & 8 & 12 & 8 & 1 \\
\hline Shipping route cross & 0 & 2 & 18 & 9 & 0 \\
\hline Ship traffic flow volume & 0 & 2 & 12 & 14 & 1 \\
\hline Obstacles to navigation & 0 & 6 & 11 & 11 & 1 \\
\hline Navigational aids & 9 & 16 & 4 & 0 & 0 \\
\hline
\end{tabular}

Note: the numbers in the table indicate the number of experts using the linguistic term to evaluate a particular factor.

TABLE 8: Initial evaluation of the selected factors by authors.

\begin{tabular}{|c|c|c|c|c|c|}
\hline \multirow{2}{*}{ Concerned objects } & \multicolumn{5}{|c|}{ Linguistic terms (for qualitative risk evaluation) } \\
\hline & $\mathrm{L}$ & ML & M & $\mathrm{LH}$ & $\mathrm{H}$ \\
\hline Wind & & & & & $\sqrt{ }$ \\
\hline Current & & & $\sqrt{ }$ & & \\
\hline Fog and visibility & & & & $\sqrt{ }$ & \\
\hline Channel width & & $\sqrt{ }$ & & & \\
\hline Channel water depth & & $\sqrt{ }$ & & & \\
\hline Shipping route cross & & & & & $\sqrt{ }$ \\
\hline Ship traffic flow volume & & & & & $\sqrt{ }$ \\
\hline Obstacles to navigation & & & & $\sqrt{ }$ & \\
\hline Navigational aids & & $\sqrt{ }$ & & & \\
\hline
\end{tabular}

Note: " $\sqrt{ }$ " indicates the authors evaluating a particular factor using the corresponding linguistic term.

TABle 9: Model results for comparison.

\begin{tabular}{lccc}
\hline Concerned objects & $\begin{array}{c}\text { RCMs using the proposed calculation } \\
\text { method }\end{array}$ & $\begin{array}{c}\text { RCMs using the simplified calculation } \\
\text { method }\end{array}$ & $\begin{array}{c}\text { Evaluation based on fuzzy } \\
\text { theory }\left(x_{\mathrm{FT}}\right)\end{array}$ \\
\hline Wind & $R(0.6391,0.1531,0.0605)$ & $R(0.6391,0.0571,0.0072)$ & 0.6391 \\
Current & $R(0.2751,0.1787,0.0811)$ & $R(0.2751,0.0698,0.0088)$ & 0.2751 \\
Fog and visibility & $R(0.7004,0.1392,0.0771)$ & $R(0.7004,0.0650,0.0082)$ & 0.7004 \\
Channel width & $R(0.4594,0.1606,0.0693)$ & $R(0.4594,0.0536,0.0068)$ & 0.4594 \\
Channel water depth & $R(0.5103,0.1729,0.0848)$ & $R(0.5612,0.0503,0.0064)$ & 0.5103 \\
Shipping route cross & $R(0.5612,0.1363,0.0325)$ & $R(0.6097,0.0566,0.0071)$ & 0.5612 \\
Ship traffic flow & $R(0.6097,0.1578,0.0396)$ & & 0.6097 \\
volume & $R(0.5549,0.1678,0.0586)$ & $R(0.5549,0.0561,0.0071)$ & 0.5549 \\
Obstacles to & $R(0.2418,0.1732,0.0613)$ & $R(0.2418,0.0724,0.0091)$ & 0.2418 \\
navigation & & & \\
Navigational aids & & & \\
\hline
\end{tabular}




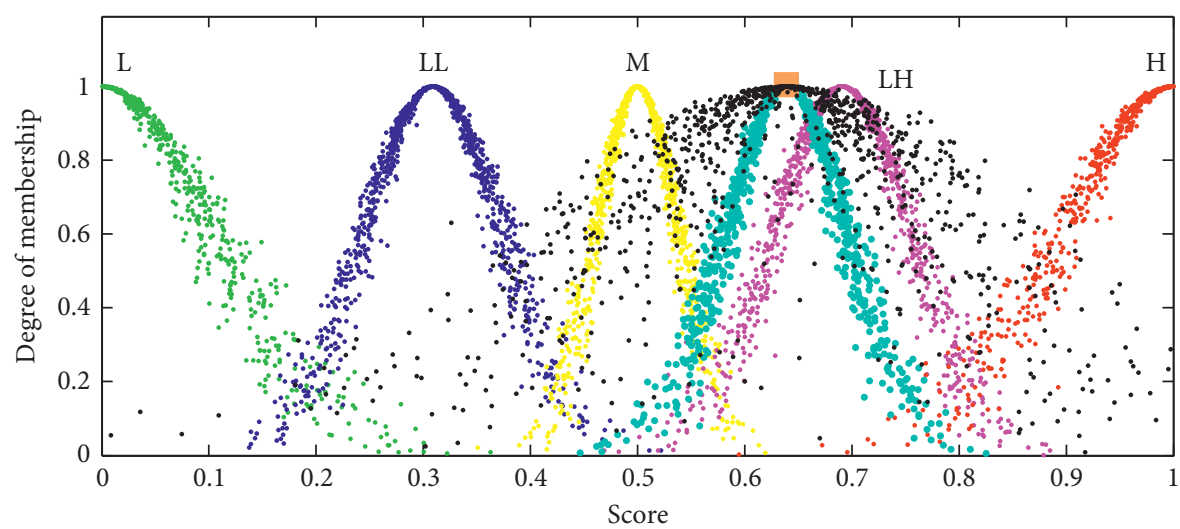

- By simplified algorithm

- By the proposed algorithm

By fuzzy evaluation

Figure 3: Plotting and comparison of model results for evaluating "wind."

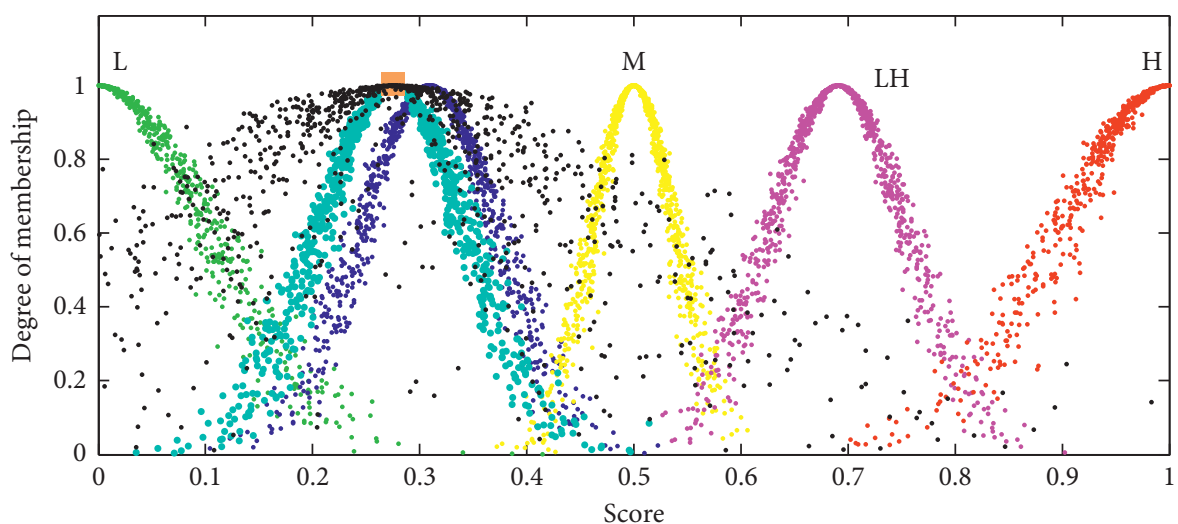

- By simplified algorithm

- By the proposed algorithm

By fuzzy evaluation

FIgURE 4: Plotting and comparison of model results for evaluating "current."

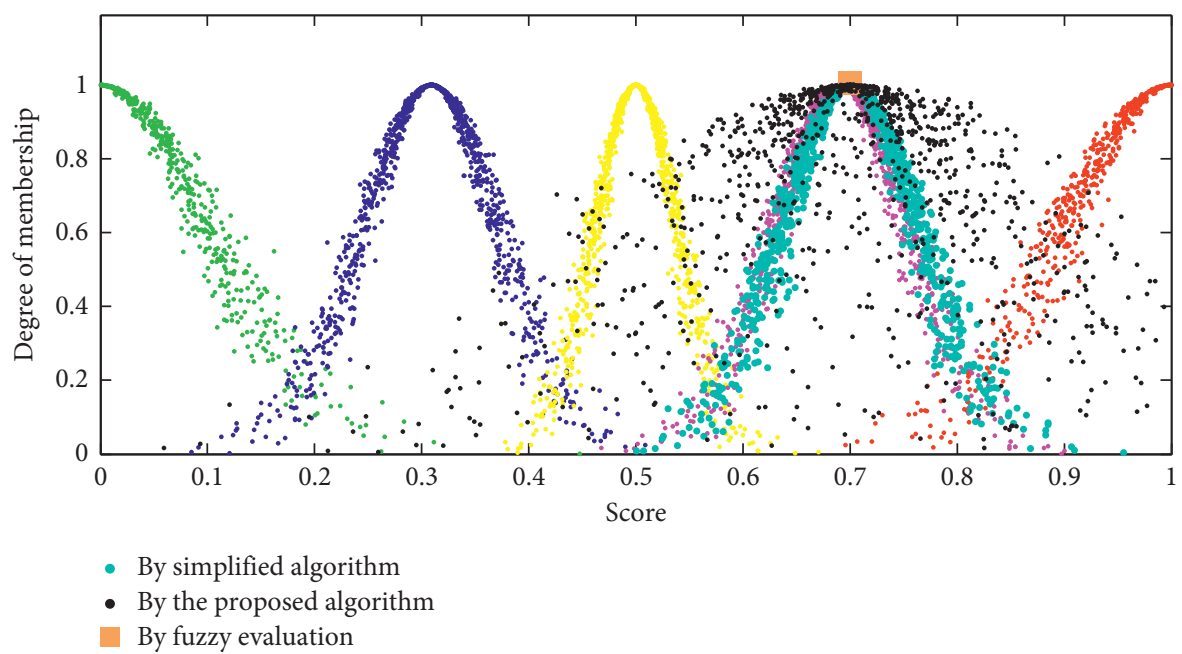

FIGURE 5: Plotting and comparison of model results for evaluating "fog and visibility." 


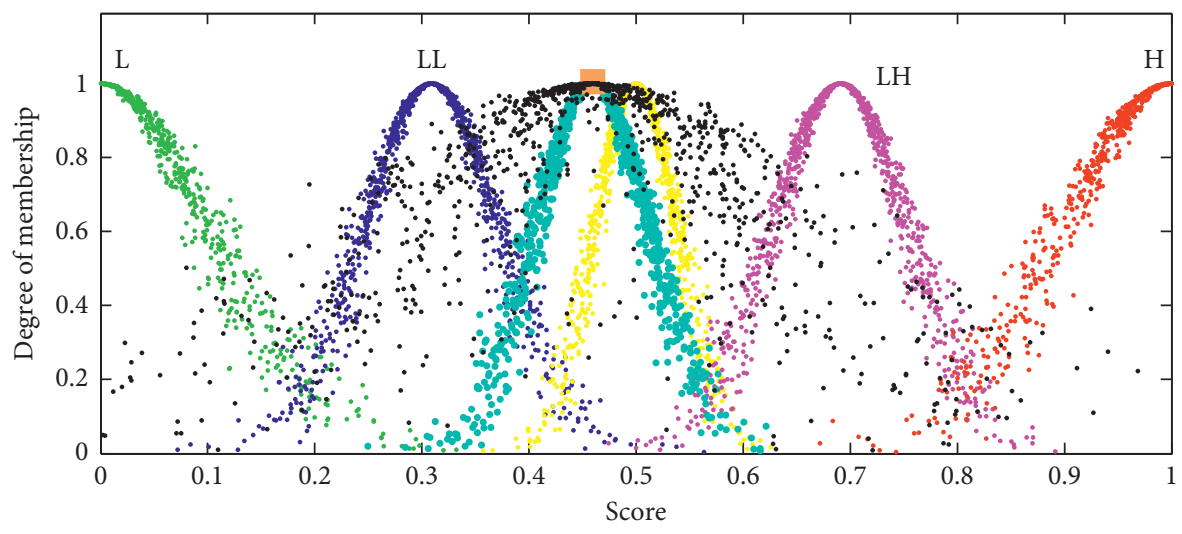

- By simplified algorithm

- By the proposed algorithm

By fuzzy evaluation

Figure 6: Plotting and comparison of model results for evaluating "channel width."

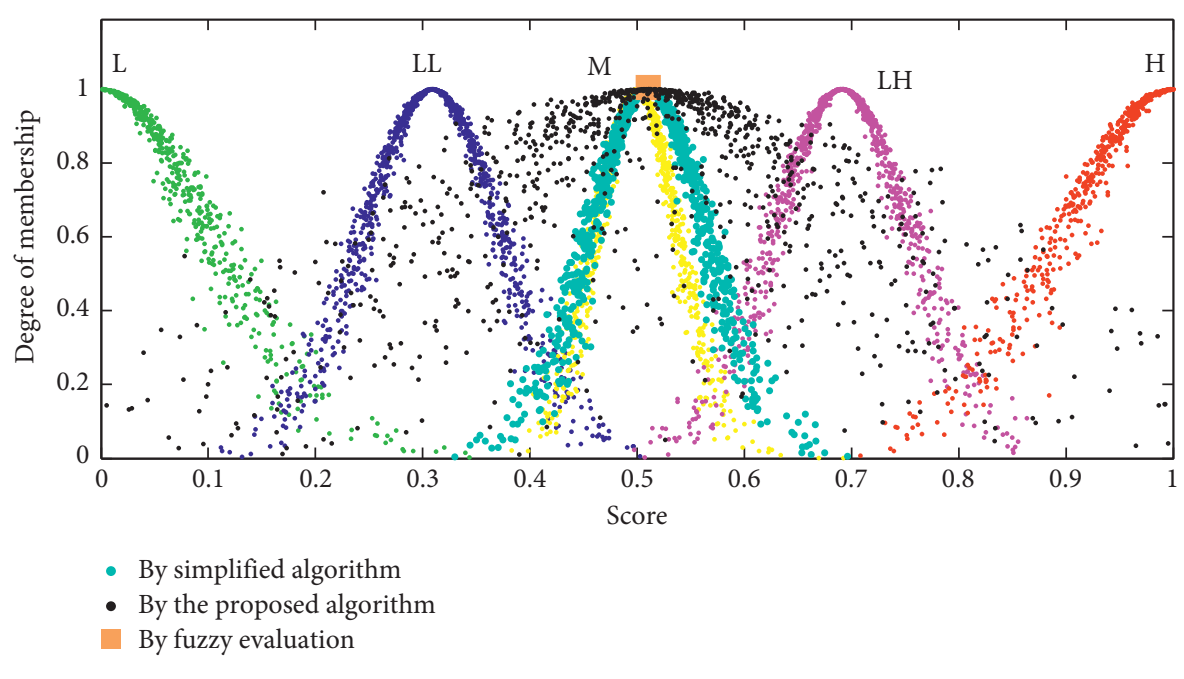

Figure 7: Plotting and comparison of model results for evaluating "channel water depth."

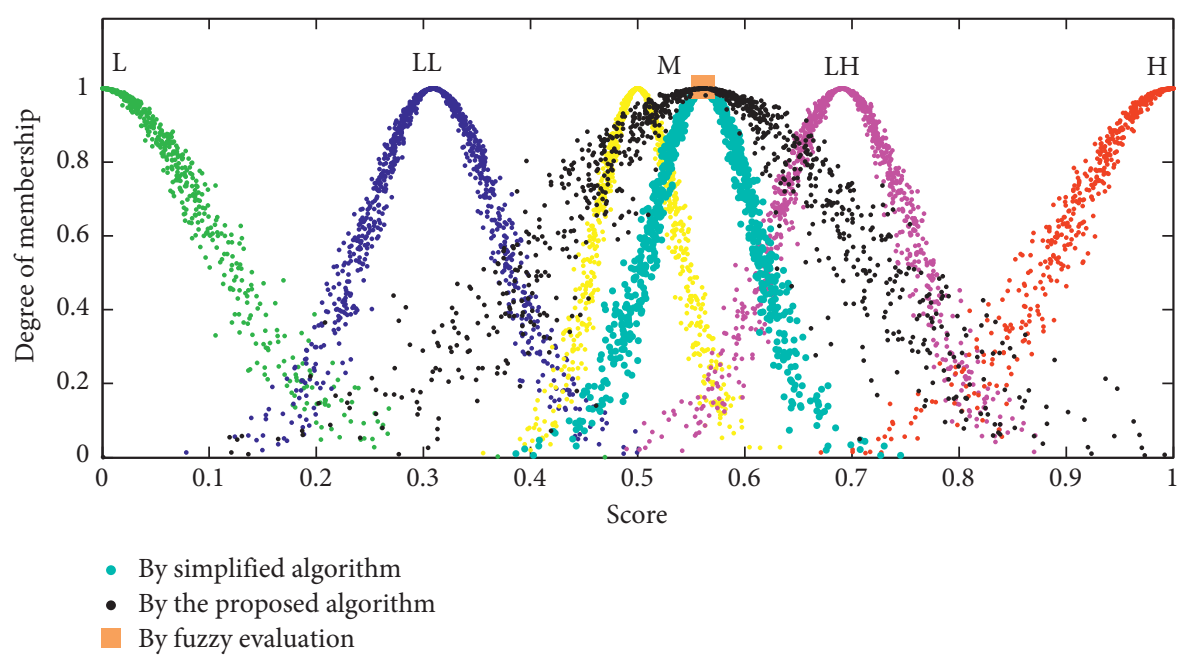

FIGURE 8: Plotting and comparison of model results for evaluating "shipping route cross." 


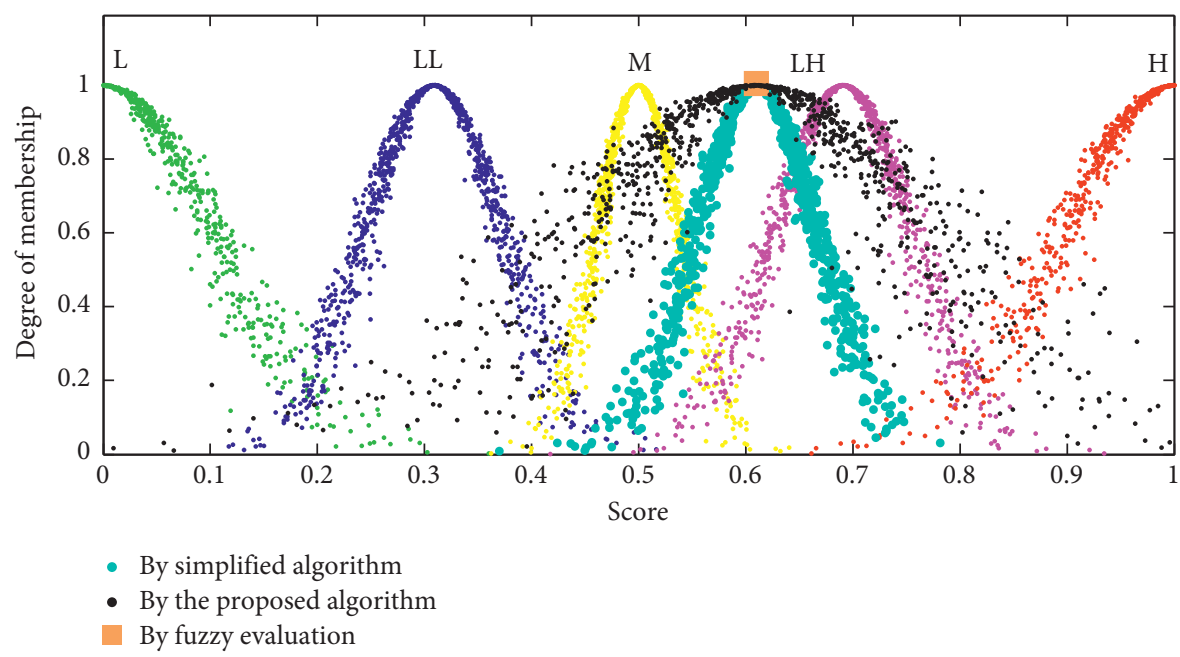

Figure 9: Plotting and comparison of model results for evaluating "ship traffic flow volume."

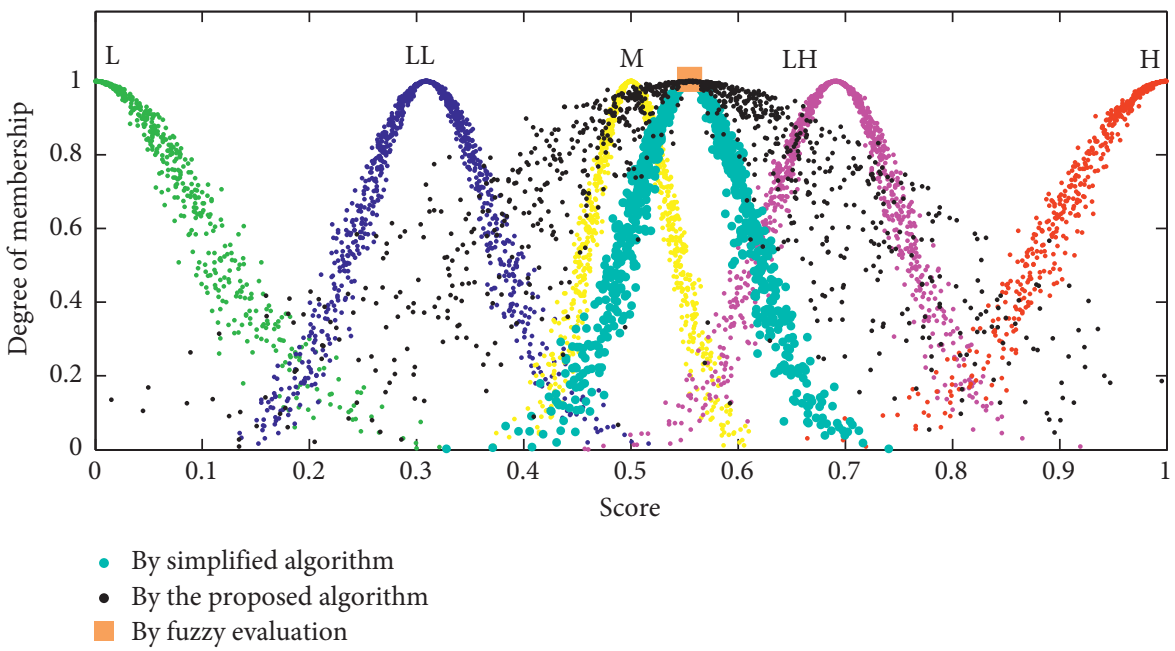

Figure 10: Plotting and comparison of model results for evaluating "obstacles to navigation."

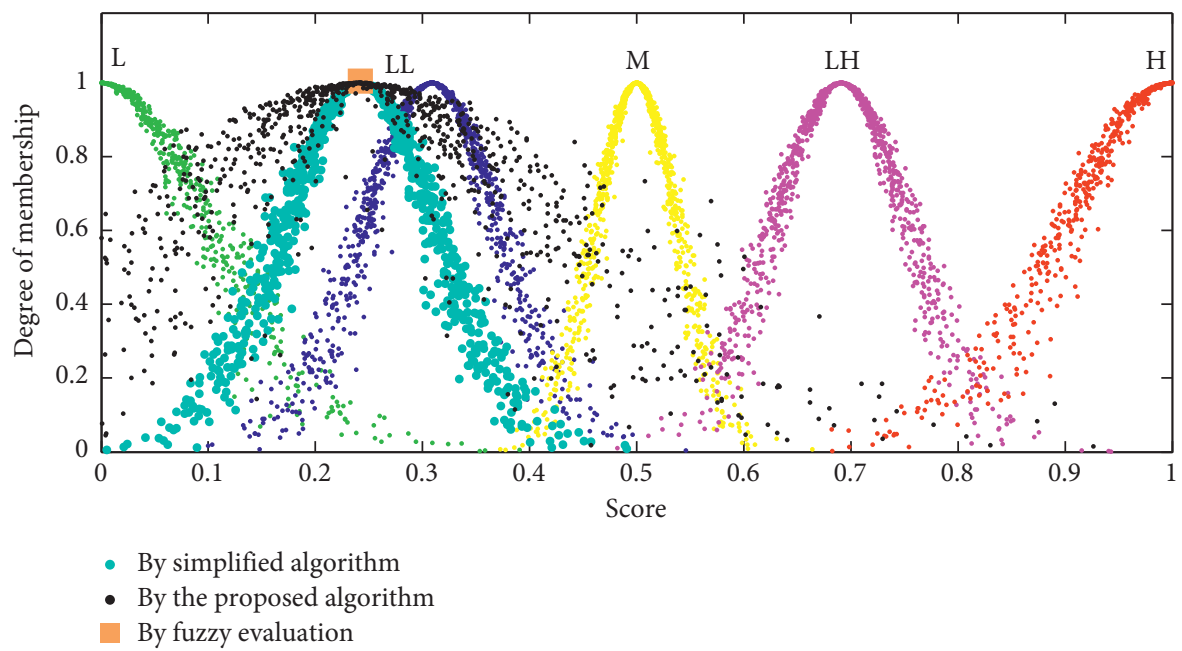

FIGURE 11: Plotting and comparison of model results for evaluating "Navigational aids." 


$$
\mu(B(k))=\frac{N_{k}}{N},
$$

where $N_{k}$ is the number of times $B(k)$ was used and $N$ is the total number of experimental runs. The initial evaluations were based on expert judgements; therefore, $N_{k}$ is the number of experts who used $B(k)$ and $N$ is the total number of experts. Table 9 (column 3 ) lists the results, and these results are plotted in Figure 3-11 (the brown square marker) for each evaluation factor.

4.6.2. Evaluation Using RCM Based on a Simplified Method. A previous study [65] used a simplified method to obtain the cloud model parameters. To convert the fuzzy evaluations of the experts into cloud models, the parameters of the benchmark cloud (entropy and hyperentropy) were directly assigned to individual clouds. Next, these individual clouds were integrated to obtain a comprehensive cloud. During this process, if an expert $i$ uses comment $j$ for the fuzzy evaluation, then the individual cloud is given by

$$
C_{j}^{i}=C_{j}^{B}
$$

where $C_{j}^{\mathrm{B}}$ is the benchmark cloud corresponding to comment $j$. The simplified algorithm was used to obtain cloud models for nine evaluation factors. The calculated parameters are listed in Table 9 (column 2), and the evaluation results are plotted in Figures 3-11 (the turquoise dots).

4.6.3. Comparative Analysis and Discussion. First, the comparative analysis was performed to prove the effectiveness of the proposed method. Table 9 presents the results of the various methods. Risk $x$ based on fuzzy theory and cloud model parameters based on the simplified algorithm agreed with the cloud model parameters of the proposed RCM method. Thus, all three methods agreed in their quantitative evaluation of the risk. However, the fuzzy theory-based method was only able to provide a single parameter $(x)$ for risk evaluation. Although it provided the most likely qualitative value that was consistent with the expected value $\left(R_{1}\right)$ of the RCM, it could not represent uncertainty characteristics such as the randomness and fuzziness. In contrast, these uncertainties were captured by the entropy $\left(R_{2}\right)$ and hyperentropy $\left(R_{3}\right)$ of the RCM-based method. In addition, the plots of the RCM droplets (Figures 3-11) intuitively expressed the risk level, which is beneficial for analysis. The cloud model based on the simplified algorithm was overly reliant on the benchmark cloud, resulting in similar entropy and hyperentropy. Hence, the diversity was lost, and the uncertainty of the original data was not reflected well by the entropy and hyperentropy. In contrast, the entropy and hyperentropy obtained with the proposed method were based on the original data, so they provided a better fit to the uncertainty.

Further, the comparative analysis was conducted to prove the sensitivity of the proposed method. Evaluations on ship route cross and obstacles to navigation were used as examples for sensitivity analysis. We supposed that D6 was the coalition of data in row 6 of both Tables 1 and 3, and D6 was used to obtain the RCM for ship route cross-evaluation. Next, we supposed that D8 was the coalition of data in row 8 of both Tables 1 and 3, and D8 was used to obtain the RCM for obstacles to navigation evaluation. Noticeably, D8 was different from D6: there were differences (inherently due to randomness of experts' judgements) between these two datasets.

We supposed that $R_{\mathrm{src}}^{p}\left(R_{1 \mathrm{src}}^{p}, R_{2 \mathrm{src}}^{p}, R_{3 \mathrm{src}}^{p}\right)$ and $R_{\mathrm{otn}}^{p}\left(R_{1 \mathrm{otn}}^{p}\right.$, $\left.R_{2 \text { otn }}^{p}, R_{3 \text { otn }}^{p}\right)$ were the RCMs for ship route cross-evaluation and obstacles to navigation using the proposed computational method. In Table 9, $\left(R_{1 \mathrm{src}}^{p}, R_{2 \mathrm{src}}^{p}, R_{3 \mathrm{src}}^{p}\right)=$ $(0.5612,0.1363,0.0325),\left(R_{1 \mathrm{otn}}^{p}, R_{2 \mathrm{otn}}^{p}, R_{3 \mathrm{otn}}^{p}\right)=(0.5549,0.16$ $78,0.0586)$. In addition, we supposed that $R_{\mathrm{src}}^{s}\left(R_{1 \mathrm{src}}^{s}, R_{2 \mathrm{src}}^{s}\right.$, $\left.R_{3 \mathrm{src}}^{s}\right)$ and $R_{\text {otn }}^{s}\left(R_{1 \text { otn }}^{s}, R_{2 \text { otn }}^{s}, R_{3 \mathrm{otn}}^{s}\right)$ were the RCMs for ship route cross-evaluation and obstacles to navigation using the simplified computational method. In Table 9, ( $R_{1 \text { src }}^{s}, R_{2 \mathrm{src}}^{s}$, $\left.R_{3 \mathrm{src}}^{s}\right)=(0.5612,0.0503,0.0064), \quad\left(R_{1 \mathrm{otn}}^{s}, R_{2 \mathrm{otn}}^{s}, R_{3 \mathrm{otn}}^{s}\right)=($ $0.5549,0.0561,0.0071)$. Furthermore, $x_{\text {stc }}^{\mathrm{FT}}$ and $x_{\mathrm{otn}}^{\mathrm{FT}}$ were supposed to be the evaluations on ship route cross-evaluation and obstacles to navigation using fuzzy theory. In Table 9, $x_{\text {stc }}^{\mathrm{FT}}=0.5612, x_{\text {otn }}^{\mathrm{FT}}=0.5549$.

The following situations were observed. When data changed, the fuzzy theory-based risk evaluation decisionmaking method using a single variable was not sensitive enough to data: it was likely to provide very similar results, slight numerical difference between $x_{\text {stc }}^{\mathrm{FT}}$ and $x_{\mathrm{otn}}^{\mathrm{FT}}$. The simplified algorithm-based RCM was not enough sensitive to data: when the data changed, parameters in the cloud model showed slight difference: slight numerical differences between $R_{1 \text { otn }}^{s}$ and $R_{1 \text { src }}^{s}, R_{2 \text { otn }}^{s}$ and $R_{2 \mathrm{src}}^{s}$, and $R_{3 \text { otn }}^{s}$ and $R_{3 \mathrm{src}}^{s}$. In contrast, when the data changed, the decision-making method based on the proposed RCM was sensitive to data for the cases of evaluations on ship route cross-evaluation and obstacles to navigation. Although $R_{\text {lotn }}^{p}$ was close to $R_{1 \text { src }}^{p}$, there were clear numerical differences between $R_{\text {2otn }}^{p}$ and $R_{2 \mathrm{src}}^{p}, R_{3 \mathrm{otn}}^{p}$ and $R_{3 \mathrm{src}}^{p}$. Corresponding to entropy and hyperentropy, the second and third parameters in the RCM were able to reflect the "uncertainty" of raw data and the final evaluations. It can be observed that cloud droplet' locations of $R_{\mathrm{otn}}^{p}\left(R_{1 \text { otn }}^{p}, R_{2 \text { otn }}^{p}, R_{3 \text { otn }}^{p}\right)$ in the frame (Figure 10) are clearly different from those of $R_{\mathrm{src}}^{p}\left(R_{1 \mathrm{src}}^{p}, R_{2 \mathrm{src}}^{p}, R_{3 \mathrm{src}}^{p}\right)$ (Figure 8): cloud droplets of $R_{\mathrm{otn}}^{p}$ are more spread out vertically and horizontally. Thus, the sensitivity of the proposed RCM allowed it to provide a differentiated evaluation conclusion using three parameters, supporting visual plots of cloud droplets.

Thus, the case study and comparative analysis demonstrated the following advantages of the proposed RCM method:

(1) The RCM method reflected the concept of multiple attribute decision-making (MADM) for risk evaluation. For example, it integrated fuzzy information from the qualitative judgement of an expert, random information from the independent judgements of many experts, and a wide range of individual comments to generate a comprehensive result.

(2) The RCM method reflected uncertainty in the risk evaluation by integrating fuzziness and randomness 
without losing knowledge-based information. In the RCM, the expected value depicted the central tendency of expert judgements, and the entropy and hyperentropy resulted in discrete locations of cloud droplets that reflected the uncertainty of the evaluation process and results.

(3) The RCM method allowed for both quantitative and qualitative risk evaluation. The characteristic parameters of RCM could be used for quantitative evaluation, and an RCM corresponded to a linguistic term for qualitative evaluation.

(4) The RCM approach provided risk evaluation-related decision-making results using both three parameters and cloud droplets' locations in the coordinates. In addition to using the first parameter to determine the central tendency, the RCM still provided decisionmaking results based on entropy and hyperentropy to determine "uncertainty" such as randomness and fuzziness. This way of decision-making made the method sensitive to data, and this is why the RCM evaluation results were more refined.

\section{Conclusion}

In this study, an RCM method for NNEt risk evaluation was proposed. The concept, form, and meanings of the RCM were described, and a stepwise computational approach based on fuzzy statistics was presented. The proposed method was validated through a case study, where it was applied to the NNEt risk evaluation of the Qiongzhou Strait. The RCMs of nine evaluation factors were obtained, and the results were compared with those obtained using a fuzzy theory-based evaluation and an RCM evaluation using an earlier simplified algorithm. The proposed RCM method was effective in providing both quantitative and qualitative results and reflected uncertainty within the risk of evaluation process. It also provided risk evaluation-related decisionmaking results using both three parameters and cloud droplets' locations in the coordinates. Consequently, this made the method sensitive to data and ensured the refinement of evaluation results.

\section{Data Availability}

The cited initial qualitative evaluations (data in Table 7) used to support the findings of this study have been deposited in the public and open publication [64], and the additional initial qualitative evaluation (data in Table 8) is originally available from the authors .

\section{Conflicts of Interest}

The authors declare no conflicts of interest regarding the publication of this paper.

\section{Acknowledgments}

Heartfelt appreciations are given to the experts for fulfilling the questionnaires, sharing their experiential knowledge and giving their evaluations. Sincere appreciations are delivered to scholars of the references for their outstanding contributions and to the reviewers and journal editors for their helpful comments, suggestions, and kind guidance. Special thanks are given to the research projects for their infrastructural and financial support. Also, the authors would like to thank Editage (http://www.editage.cn) for English language editing. This paper was supported by the (1) Open Fund of Hubei Key Laboratory of Inland Shipping Technology (Grant no. NHHY2019002), (2) Fund of Bureau of Science and Technology of Zhoushan City (Grant no. 2020C21024), and (3) National Key R\&D Program of China (Grant nos. 2018YFC1407400/03 and 2018YFC0810400/05).

\section{References}

[1] Y.-b. Cheng, X.-h. Chen, H.-l. Li et al., "Analysis and comparison of Bayesian methods for measurement uncertainty evaluation," Mathematical Problems in Engineering, vol. 2018, Article ID 7509046, 10 pages, 2018.

[2] C. Zhen-Song, Z. Xuan, M. R. Rosa et al., "Heterogeneous interrelationships among attributes in multi-attribute decision-making: an empirical analysis," International Journal of Computational Intelligence Systems, vol. 12, no. 2, pp. 984997, 2019.

[3] Z.-S. Chen, L. Martínez, K.-S. Chin, and K.-L. Tsui, "Twostage aggregation paradigm for HFLTS possibility distributions: a hierarchical clustering perspective," Expert Systems with Applications, vol. 104, pp. 43-66, 2018.

[4] Z. Chen, M. Li, W. Kong et al., "Evaluation and selection of HazMat transportation alternatives: a PHFLTS- and TOPSISintegrated multi-perspective approach," vol. 16, no. 21, p. 4116, 2019.

[5] Z.-S. Chen, X. Zhang, K. Govindan, X.-J. Wang, and K.-S. Chin, "Third-party reverse logistics provider selection: a computational semantic analysis-based multi-perspective multi-attribute decision-making approach," Expert Systems with Applications, vol. 166, Article ID 114051, 2021.

[6] W.-M. Wey, "Constructing urban dynamic transportation planning strategies for improving quality of life and urban sustainability under emerging growth management principles," Sustainable Cities and Society, vol. 44, pp. 275-290, 2019.

[7] N. P. Ventikos, D. I. Stavrou, and A. Andritsopoulos, "Studying the marine accidents of the Aegean Sea: critical review, analysis and results," Journal of Marine Engineering \& Technology, vol. 16, no. 3, pp. 103-113, 2017.

[8] L. Cao, Z. Liu, and S. Zhang, "Research of quantitative marine individual risk model based on probability theory," in Proceedings of 2014 5th International Conference on Intelligent Systems Design and Engineering Applications (ISDEA 2014), pp. 781-784, Hunan, China, June 2014.

[9] C. Mabrouki, F. Bentaleb, and A. Mousrij, "A decision support methodology for risk management within a port terminal," Safety Science, vol. 63, pp. 124-132, 2014.

[10] H. S. Sii, T. Ruxton, and J. Wang, "A fuzzy-logic-based approach to qualitative safety modelling for marine systems," Reliability Engineering \& System Safety, vol. 73, no. 1, pp. 19-34, 2001.

[11] J.-Y. Pak, G.-T. Yeo, S.-W. Oh, and Z. Yang, "Port safety evaluation from a captain's perspective: the Korean experience," Safety Science, vol. 72, pp. 172-181, 2015. 
[12] Y. Tian, X. Sun, L. Chen, and L. Huang, "Risk assessment of nautical navigational environment based on grey fixed weight cluster," Promet-Traffic \& Transportation, vol. 29, no. 3, pp. 331-342, 2017.

[13] K. Liu, J. Zhang, X. Yan, Y. Liu, D. Zhang, and W. Hu, "Safety assessment for inland waterway transportation with an extended fuzzy TOPSIS," Proceedings of the Institution of $\mathrm{Me}$ chanical Engineers, Part O: Journal of Risk and Reliability, vol. 230, no. 3, pp. 323-333, 2016.

[14] B. Wu, Y. Wang, J. Zhang, E. E. Savan, and X. Yan, "Effectiveness of maritime safety control in different navigation zones using a spatial sequential DEA model: Yangtze River case," Accident Analysis \& Prevention, vol. 81, pp. 232-242, 2015.

[15] D. Y. Li, H. J. Meng, and X. M. Shi, "Membership cloud and cloud generator," Computer Research and Development, vol. 32, no. 6, pp. 16-18, 1995.

[16] D. Li, D. Cheung, X. Shi, and V. Ng, "Uncertainty reasoning based on cloud models in controllers," Computers \& Mathematics with Applications, vol. 35, no. 3, pp. 99-123, 1998.

[17] X. Y. Du, Q. J. Yi, K. L. Huang et al., "Qualitative quantitative transformation method and application based on cloud model," Engineering Systems and Electronics, vol. 30, no. 4, pp. 772-776, 2008.

[18] M. Wang, X. Wang, Q. Liu, F. Shen, and J. Jin, “A novel multidimensional cloud model coupled with connection numbers theory for evaluation of slope stability," Applied Mathematical Modelling, vol. 77, pp. 426-438, 2020.

[19] D. Song, M. Yang, H. Bai et al., "A comprehensive evaluation model of power market operation efficiency based on extension cloud theory," Energy Procedia, vol. 156, pp. 302-309, 2019.

[20] J. Li, H. Fang, and W. Song, "Modified failure mode and effects analysis under uncertainty: a rough cloud theory-based approach," Applied Soft Computing, vol. 78, pp. 195-208, 2019.

[21] S. Lou, Y. Feng, Z. Li et al., "An integrated decision-making method for product design scheme evaluation based on cloud model and EEG data," Advanced Engineering Informatics, vol. 43, pp. 1-9, 2020.

[22] Q. Liu, M. Wang, T. Zhou, F. Shen, and J. Jin, “A connection cloud model coupled with extenics for water eutrophication evaluation," Earth Science Informatics, vol. 12, no. 4, pp. 659-669, 2019.

[23] X. Lv, C. Chen, P. Wang et al., "Status evaluation of mobile welding robot driven by fuel cell hybrid power system based on cloud model," Energy Conversion and Management, vol. 198, pp. 1-18, 2019.

[24] L. Yang, R. Zhang, T. Hou, Z. Hao, and J. Liu, "Hesitant cloud model and its application in the risk assessment of "the twenty-first Century maritime Silk Road," Mathematical Problems in Engineering, vol. 2016, Article ID 5620803, 11 pages, 2016.

[25] J. Wang, W. J. Xiao, and S. W. Wang, "An improved effectiveness evaluation method based on cloud model," Fire Control \& Command Control, vol. 35, no. 3, pp. 97-99, 2010.

[26] J. Q. Wang and T. Liu, "Uncertain language evaluation Based on comprehensive cloud," Control and Decision, vol. 27, no. 8, pp. 185-190, 2012.

[27] Z.-S. Chen, K.-S. Chin, L. Martínez, and K.-L. Tsui, "Customizing semantics for individuals with attitudinal HFLTS possibility distributions," IEEE Transactions on Fuzzy Systems, vol. 26, no. 6, pp. 3452-3466, 2018.
[28] Z.-S. Chen, M. Xu, X.-J. Wang, K.-S. Chin, K.-L. Tsui, and L. Martinez, "Individual semantics Building for HFLTS possibility distribution with applications in domain-specific collaborative decision making," IEEE Access, vol. 6, pp. 78803-78828, 2018.

[29] S.-H. Xiong, Z.-S. Chen, and K.-S. Chin, "A novel MAGDM approach with proportional hesitant fuzzy sets," International Journal of Computational Intelligence Systems, vol. 11, no. 1, pp. 256-271, 2018.

[30] B. Farhadinia, "Multiple criteria decision-making methods with completely unknown weights in hesitant fuzzy linguistic term setting," Knowledge-Based Systems, vol. 93, pp. 135-144, 2016.

[31] H. Z. Chen, L. B. Yang, S. W. Wang et al., "High-risk waters identification and evaluation in China coasts," Journal of Dalian Maritime University, vol. 36, no. 2, pp. 42-46, 2010.

[32] S. P. Hu, Q. G. Fang, and J. P. Zhang, "The coastal water traffic safety risk assessment studies," Navigation of China, vol. 33, no. 1, pp. 50-55, 2010.

[33] S. Y. Xuan, F. M. Li, S. P. Hu et al., "The ship navigation risk assessment in Guangzhou port waters," Navigation of China, vol. 36, no. 4, pp. 96-99, 2013.

[34] F. Lu, W. Li, and H. Zhang, "Safety assessment of ship anchoring based on the extenics," Journal of Dalian Maritime University, vol. 41, no. 1, pp. 10-14, 2015.

[35] D. Y. Li and C. Y. Liu, "The universality of normal cloud," Chinese Engineering Science, vol. 6, no. 8, pp. 28-34, 2004.

[36] K. C. Tseng, C. S. Hwang, and Y. C. Su, "Using cloud model for default voting in collaborative filtering," Journal of Convergence Information Technology, vol. 6, pp. 68-74, 2011.

[37] K. C. Di, D. R. Li, and D. Y. Li, "The cloud theory application in spatial data mining and knowledge discovery," Journal of Image and Graphics, vol. 4, no. 11, pp. 929-935, 1999.

[38] C. Sun, D. R. Nie, J. P. Zhang et al., "Risk assessment of navigation environment of Waigaoqiao waters," Navigation of China, vol. 35, no. 2, pp. 68-71, 2012.

[39] H. Chen, B. S. Liu, G. L. Wang et al., "Comprehensive risk assessment of shipping environment in Luoyuan bay," Navigation of China, vol. 36, no. 4, pp. 86-91, 2013.

[40] B. Wu, T. L. Yip, L. Xie, and Y. Wang, “A fuzzy-MADM based approach for site selection of offshore wind farm in busy waterways in China," Ocean Engineering, vol. 168, pp. 121132, 2018.

[41] J. Zhang, Â. P. Teixeira, C. Guedes Soares, X. Yan, and K. Liu, "Maritime transportation risk assessment of tianjin port with Bayesian Belief networks," Risk Analysis, vol. 36, no. 6, pp. 1171-1187, 2016.

[42] M.-G. Jeong, E.-B. Lee, M. Lee, and J.-Y. Jung, "Multi-criteria route planning with risk contour map for smart navigation," Ocean Engineering, vol. 172, pp. 72-85, 2019.

[43] B. Sahin and S. Kum, "Risk assessment of arctic navigation by using improved fuzzy-AHP approach," International Journal of Maritime Engineering, vol. 157, no. 4, pp. 241-250, 2015.

[44] T. G. Fowler and E. Sorgard, "Modeling ship transportation risk," Risk Analysis, vol. 20, no. 2, pp. 225-244, 2000.

[45] J. Wang, M. Li, Y. Liu, H. Zhang, W. Zou, and L. Cheng, "Safety assessment of shipping routes in the South China Sea based on the fuzzy analytic hierarchy process," Safety Science, vol. 62, pp. 46-57, 2014.

[46] J.-F. Balmat, F. Lafont, R. Maifret, and N. Pessel, "MAritime RISk Assessment (MARISA), a fuzzy approach to define an individual ship risk factor," Ocean Engineering, vol. 36, no. 1516, pp. 1278-1286, 2009. 
[47] T. Liu, "Navigation risk analysis and countermeasures of Rongjiang waterway in east Guangdong province," Marine Technology, no. 5, pp. 26-30, 2019.

[48] Q. Liu, D. D. Han, Y. Q. Chen et al., "Research on navigation risk evolution in Three Gorges dam area based on system dynamics," China Safety Science Journal, vol. 26, no. 4, pp. 19-23, 2016.

[49] X. L. Nie, D. Ran, and X. W. Yue, "Risk assessment of navigation environment based on fuzzy comprehensive evaluation," Journal of Dalian Maritime University, vol. 39, no. 1, pp. 27-30, 2013.

[50] M. J. Li and C. W. Mei, "On assessment of marine traffic safety of Jiaxing port based on fuzzy comprehensive evaluation," Science \& Technology Vision, no. 34, pp. 43-45, 2012.

[51] M. J. Shao, "Research of image segmentation algorithm based on the backward cloud model," Fire Control \& Command Control, vol. 35, no. 7, pp. 139-142, 2010.

[52] H. Chen and B. Li, "Data fitting based backward cloud algorithm with certainty degree," Application Research of Computers, vol. 33, no. 12, pp. 3663-3666, 2016.

[53] H. J. Liu, H. L. Li, D. Y. Li et al., "The application of reverse cloud in the qualitative evaluation," Chinese Journal of Computers, vol. 26, no. 8, pp. 1009-1014, 2003.

[54] Q. Wang, X. Wang, L. Xie, Q. Shang, and Y. Lü, “Observed water current and transport through Qiongzhou Strait during august 2010," Chinese Journal of Oceanology and Limnology, vol. 32, no. 3, pp. 703-708, 2014.

[55] C. Chen, P. Li, M. Shi, J. Zuo, M. Chen, and H. Sun, "Numerical study of the tides and residual currents in the Qiongzhou Strait," Chinese Journal of Oceanology and Limnology, vol. 27, no. 4, pp. 931-942, 2009.

[56] M. Shi, C. Chen, Q. Xu et al., "The role of Qiongzhou Strait in the seasonal variation of the South China sea circulation," Journal of Physical Oceanography, vol. 32, no. 1, pp. 103-121, 2002.

[57] X. C. Xu, J. W. Xin, G. F. Liang et al., "Observation and analysis OF sea surface wind over the Qiongzhou Strait," Journal of Tropical Meteorology, vol. 27, no. 1, pp. 118-124, 2011.

[58] J. Huang, "Comments on the oil spill emergency response ability of Qiongzhou Strait," World Shipping, vol. 36, no. 3, pp. 15-17, 2013.

[59] T. Li, A Study on the Problems of Qiongzhou Strait Traffic Management and Countermeasures, Wuhan University of Technology, Wuhan, China, 2013.

[60] Z. M. Liu and Z. Kang, "Safety control of ships navigating in the Qiongzhou Strait in foggy weather," China Marine Safety, no. 2, pp. 28-30, 2011.

[61] W. H. Zhu, "The role of VTS in the safety of ship navigation in the Qiongzhou Strait," China Water Transport, vol. 9, no. 8, pp. 58-59, 2009.

[62] H. H. Dong and J. Ye, "Navigation safety in Qiongzhou Strait waters," Pearl River Water Transport, no. 23, pp. 64-65, 2013.

[63] S. K. Huang, "Discussion on navigation safety in fog at north part of Qiongzhou Strait," Consume Guide, no. 4, p. 236, 2008.

[64] X. C. Sun, "Qiongzhou strait shipping safety evaluation studies," Guangzhou Maritime College Journal, vol. 22, no. 57, pp. 62-64, 2014.

[65] Y.-F. Tian, L.-J. Chen, L.-W. Huang, and J.-M. Mou, "Featured risk evaluation of nautical navigational environment using a risk cloud model," Journal of Marine Engineering \& Technology, vol. 19, no. 3, pp. 115-129, 2020. 